\title{
SNPs and interaction analyses of IRF6, MSX1 and PAX9 genes in patients with non-syndromic cleft lip with or without palate
}

\author{
TAO SONG, DI WU, YONGQIAN WANG, HAIDONG LI, NINGBEI YIN and ZHENMIN ZHAO
}

Center of Cleft Lip and Palate, Plastic Surgery Hospital,

Chinese Academy of Medical Sciences and Peking Union Medical College, Shijingshan, Beijing 100144, P.R. China

Received March 31, 2013; Accepted July 23, 2013

DOI: $10.3892 / \mathrm{mmr} .2013 .1617$

\begin{abstract}
Non-syndromic cleft lip with or without cleft palate (NSCL/P) is a common congenital deformity, often associated with missing or deformed teeth. The genes interferon regulatory factor 6 (IRF6), muscle segment homeobox 1 (MSX1) and paired box gene 9 (PAX9) are important for the development of the maxillofacial region and dentition. The aim of this study was to explore how genetic variations in IRF6, MSX1 and PAX9, as well as gene-gene interactions, are associated with NSCL/P. We investigated 9 IRF6 tag single nucleotide polymorphisms (SNPs), 2 MSX1 tag SNPs and 8 PAX9 tag SNPs selected from HapMap data from the Chinese population. The SNPs were examined for associations with NSCL/P in 204 patients and 226 controls. The results demonstrated a significant association between NSCL/P and rs2073485, rs2235371, rs2236909 and rs861020 in the IRF6 gene, and haplotype analysis supported these findings. A marginally significant difference was observed in the NSCL/P group for rs17176643 in the PAX9 gene compared to the control group. The results of gene-gene interaction analyses also indicated that the combination of rs2073485, rs2235371 or rs2236909 in IRF6 and rs17176643 in PAX9, increased the risk of NSCL/P. Thus, our study provided additional understanding of IRF6 variations in patients with NSCL/P and suggested that
\end{abstract}

Correspondence to: Dr Ningbei Yin or Dr Zhenmin Zhao, Center of Cleft Lip and Palate, Plastic Surgery Hospital, Chinese Academy of Medical Sciences and Peking Union Medical College, 33 Badachu Road, Shijingshan, Beijing 100144, P.R. China

E-mail: yinningbei@yahoo.com.cn

E-mail: zhaozhenmin0098@vip.sina.com

Abbreviations: NSCL/P, non-syndromic cleft lip with or without cleft palate; IRF6, interferon regulatory factor 6; MSX1, muscle segment homeobox 1; PAX9, paired box gene 9; HWE, Hardy-Weinberg equilibrium; LD, linkage disequilibrium; MAF, minor allele frequency; OR, odds radio; CI, confidence interval

Key words: non-syndromic cleft lip with or without cleft palate, interferon regulatory factor 6 , muscle segment homeobox 1 , paired box gene 9 interactions between the IRF6 and PAX9 genes are potentially important for susceptibility to NSCL/P.

\section{Introduction}

Non-syndromic cleft lip with or without cleft palate (NSCL/P) is a complex disorder with a multifactorial etiology involving genetic and environmental factors. Through the use of a wide range of genetic approaches, various candidate genes and chromosomal regions associated with NSCL/P have been identified (1). However, these findings remain controversial, due in part to phenomena such as genetic heterogeneity and incomplete dominance.

Interferon regulatory factor 6 (IRF6) is a key factor involved in the development of the maxillofacial region and teeth. Several studies have suggested that variations in IRF6 may be important in the etiology of NSCL/P (2). The SNP rs2235371 (V274I) was the first marker in IRF6 shown to be associated with NSCL/P, notably in Asian and South American populations (3). This association was subsequently identified in additional populations (4-7). Several genetic markers in IRF6 have also demonstrated linkage and linkage disequilibrium (LD) in studies of non-syndromic oral clefts. However, there are inconsistencies across these studies $(8,9)$.

The MSX1 gene, located on human chromosome 4p16, is a member of the MSX gene family, and is crucial in the development of teeth and the craniofacial skeleton. Knock-out mouse models have shown that cleft palate results from the complete loss of MSX1 (10), with 39\% of these mutants exhibiting unilateral or bilateral cleft lip. Linkage studies have also demonstrated that the region of chromosome 4 containing MSX1, may have a causal mutation (11), and several studies have indicated an association between the MSX1 gene and oral clefts in a variety of populations (12-14).

The transcription factor PAX9 has also been shown to have a direct relationship with craniofacial development, particularly the formation of the palate and teeth. Notably, PAX9 and MSX1 are coexpressed during craniofacial development (15), and mice that are homozygous mutant for either one of these genes exhibit cleft palate and an early arrest of tooth formation. The combination of PAX9 and MSX1 nullizygosity generates a cleft lip phenotype that results from interactions between these loci (11). In addition, in a Japanese family a heterozygous missense mutation was identified in exon 3 of 
PAX9 in two siblings with NSCL/P and their phenotypically normal mother (16). Moreover, Lee et al recently demonstrated that PAX9 contributes to the risk of NSCL/P in a Korean population (17). However, there are some inconsistencies across studies in Singaporean, Taiwanese and Korean populations (18).

The abovementioned studies suggest that IRF6, MSX1 and PAX9 are crucial for the development of dentition and the maxillofacial region (19). Notably, NSCL/P is often associated with missing teeth, and recent analyses have indicated that the incidence of missing teeth is significantly higher outside the cleft region and in the mandible (20). However, whether any interactions among the three genes are associated with NSCL/P remains to be clarified. The aim of the present study was to determine the genetic variations in IRF6, MSX1 and PAX9, and to use the data from case-control studies to clarify the association of single nucleotide polymorphisms (SNPs), haplotypes and gene-gene interactions with the risk of NSCL/P.

\section{Materials and methods}

Study population. This case-control study included 204 patients with NSCL/P and 226 normal controls; the control group had no congenital malformations of the body, no family history of genetic disease, were born in the same region as the patients with NSCL/P and had a male:female ratio as close to the NSCL/P group as possible. The study participants were recruited between January, 2010 and January, 2012 from the Department of Cleft Lip and Palate, Plastic Surgery Hospital, Chinese Academy of Medical Sciences (Beijing, China). Informed consent was obtained from each participant prior to enrollment in the study, which was approved by the local ethics committee of Department of Cleft Lip and Palate, Plastic Surgery Hospital, Chinese Academy of Medical Sciences (Beijing, China). General characteristics including age, gender, ethnicity, health status and birth defects, were recorded.

SNP identification and selection. Using the HapMap genome browser (http://www.hapmap.org/cgi-perl/gbrowse/ hapmap3r2_B36) based on Han Chinese individuals from Beijing, China (CHB), 9 tag SNPs ( $\mathrm{r}^{2}$ coefficient cut-off of 0.80 with a minor allele frequency of 0.05 ), were selected to capture the IRF6 region of chromosome 1. Two tag SNPs were selected for the MSX1 region of chromosome 4 and 8 tag SNPs for the PAX9 region of chromosome 4 .

Genotype. Peripheral blood samples $(2 \mathrm{ml})$ were collected from each participant and frozen. Genomic DNA was extracted from a $200 \mu 1$ aliquot of each sample using a Tiangen $^{\mathrm{TM}}$ Genomic DNA Kit (Tiangen Biotech Co., Ltd., Haidian, China) according to the manufacturer's instructions, and stored at $-70^{\circ} \mathrm{C}$. The SNPs were genotyped using the Sequenom MassARRAY matrix-assisted laser desorption/ionization time-of-flight mass spectrometry analyzer (Sequenom Inc., San Diego, CA, USA). Primers were designed using a semi-automated method (Assay Design 3.1, Sequenom Inc.); primer sequences are available on request. The call rate for each assay was set at $>90 \%$.
Statistical analysis. Data from the control and NSCL/P groups were compared and analyzed with SPSS software, version 17.0 (SPSS Inc., Chicago, IL, USA) and Hardy-Weinberg equilibrium (HWE) software. Based on the multivariate logistic regression method, the case-control association of genotypes in three inheritance models (dominant, recessive and additive) were tested, and these models were coded as follows for genotypes $\mathrm{AA}, \mathrm{AB}$ and $\mathrm{BB}$ (assuming $\mathrm{B}$ is the minor allele): Dominant 0,1 and 1 (AA vs. $A B+B B$ ), recessive 0,0 and 1 $(\mathrm{AA}+\mathrm{AB}$ vs. $\mathrm{BB}$ ) and additive 0,1 and 2 (trend test on $\mathrm{B}$ allele count). The odds ratio (OR) and $95 \%$ confidence intervals (CI) were calculated. The association between each SNP and haplotype with NSCL/P was estimated using Haploview software (http://www.broad.mit.edu/mpg/haploview/).

For haplotype construction, genotypic data from the case and control groups was used to estimate intermarker linkage disequilibrium (LD) by measuring pairwise $\mathrm{D}^{\prime}$ and $\mathrm{r}^{2}$ and defining LD blocks. The CI method was employed in the Haploview software to define an LD block with an extended spine if $\mathrm{r}^{2}=0.8$. P-values were corrected for multiple tests with 10,000 permutations. To identify gene-gene interactions in our samples, logistic regression analysis was used to calculate ORs and 95\% CIs to search for gene-gene interactions in the NSCL/P and control groups. Interactions between SNPs were classified in four groups using a dominant model. In each case, the group carrying the least risk served as a reference, i.e., if the variant (B) was considered a risk factor, wild type (AA) served as a reference, while if the variant (B) was considered a protective factor, $\mathrm{AB}+\mathrm{BB}$ served as a reference. Bonferroni corrections for multiple SNPs were performed, and $\mathrm{P}<0.05$ and 0.019 were regarded as statistically significant.

\section{Results}

Characteristics of study subjects. A total of 19 SNPs were genotyped from the 204 patients with NSCL/P and 226 normal controls of Han Chinese origin. The mean age was $3.23 \pm 0.91$ years and the male:female ratio was 1:0.76 in the NSCL/P group. The mean age was $3.96 \pm 1.03$ years and the male:female ratio was 1:0.70 in the control group. The genomic position, nucleic acid composition and minor allele frequencies of the SNPs are summarized in Table I. We calculated the HWE for all the SNPs; none of the SNPs deviated from the HWE among these groups.

Single SNP analysis. To evaluate the association between genetic variants and the risk of NSCL/P, we compared IRF6, MSX1 and PAX9 genotype frequency distributions in the NSCL/P and control groups (Table II). For IRF6, there was a significant difference in the allele frequency of rs 2073485 , rs2235371, rs2236909 and rs861020 between the NSCL/P and control groups after correction with 10,000 permutations $(\mathrm{P}=0.0263,0.0232,0.0239$ and 0.0104 , respectively). The association between the dominant model of rs2073485, rs2235371 and rs 861020 with NSCL/P remained significant $(\mathrm{P}=0.002$, 0.002 and 0.007 , respectively). The distribution of the recessive model of rs2236909 and rs861020 differed significantly between the NSCL/P and control groups $(\mathrm{P}=0.000$ and 0.009 , respectively). Cochran-Armitage trend tests of rs2073485, rs2235371, rs2236909 and rs861020 showed a significant 
Table I. Characteristics of the polymorphisms of IRF6, MSX1 and PAX9 genes.

\begin{tabular}{|c|c|c|c|c|c|c|c|}
\hline \multirow{2}{*}{$\begin{array}{l}\text { Gene symbol } \\
\text { IRF6 }\end{array}$} & \multirow{2}{*}{$\begin{array}{c}\text { rs no. } \\
\text { rs } 17317411\end{array}$} & \multirow{2}{*}{$\frac{\text { SNP function }}{\text { UTR-3 }}$} & \multicolumn{2}{|c|}{ Location } & \multirow{2}{*}{$\frac{\text { Alleles }^{\mathrm{a}}}{\mathrm{c} / \mathrm{T}}$} & \multirow{2}{*}{$\frac{\text { MAF }}{0.042}$} & \multirow{2}{*}{$\begin{array}{c}\text { P-HWE } \\
0.629242\end{array}$} \\
\hline & & & Chr1 & 209961314 & & & \\
\hline & rs2073485 & Intron & Chr1 & 209962794 & $\mathrm{a} / \mathrm{G}$ & 0.376 & 0.597578 \\
\hline & rs2235371 & Missense & Chr1 & 209964080 & $\mathrm{C} / \mathrm{t}$ & 0.310 & 0.701308 \\
\hline & rs2013196 & Intron & Chr1 & 209968411 & $\mathrm{C} / \mathrm{t}$ & 0.123 & 0.744881 \\
\hline & rs7552506 & Intron & Chr1 & 209969902 & $\mathrm{c} / \mathrm{G}$ & 0.188 & 0.609989 \\
\hline & rs2236909 & Intron & Chr1 & 209971655 & $\mathrm{a} / \mathrm{G}$ & 0.497 & 0.133192 \\
\hline & rs861019 & UTR-5 & Chr1 & 209975386 & $\mathrm{~A} / \mathrm{g}$ & 0.257 & 0.122725 \\
\hline & rs861020 & Intron & Chr1 & 209977111 & $\mathrm{a} / \mathrm{G}$ & 0.240 & 0.052702 \\
\hline & rs3753518 & Intron & Chr1 & 209977964 & $\mathrm{C} / \mathrm{g}$ & 0.066 & 0.329238 \\
\hline \multirow[t]{2}{*}{ MSX1 } & rs1042484 & Intron & Chr4 & 4864381 & $\mathrm{c} / \mathrm{T}$ & 0.057 & 0.879223 \\
\hline & rs12532 & UTR-3 & Chr4 & 4865146 & $\mathrm{a} / \mathrm{G}$ & 0.376 & 0.441807 \\
\hline \multirow[t]{8}{*}{ PAX9 } & rs2236007 & Intron & Chr14 & 37132769 & $\mathrm{a} / \mathrm{G}$ & 0.270 & 0.479949 \\
\hline & rs2295218 & Intron & Chr14 & 37134491 & $\mathrm{a} / \mathrm{T}$ & 0.468 & 0.856159 \\
\hline & rs8004560 & Intron & Chr14 & 37140781 & $\mathrm{a} / \mathrm{G}$ & 0.255 & 0.167655 \\
\hline & rs17104928 & Intron & Chr14 & 37142554 & $\mathrm{a} / \mathrm{G}$ & 0.415 & 0.220053 \\
\hline & rs17176643 & Intron & Chr14 & 37142969 & $\mathrm{a} / \mathrm{C}$ & 0.498 & 0.311689 \\
\hline & rs11156926 & Intron & Chr14 & 37143237 & $\mathrm{a} / \mathrm{T}$ & 0.271 & 0.957203 \\
\hline & rs10131337 & Intron & Chr14 & 37144516 & $\mathrm{C} / \mathrm{t}$ & 0.297 & 0.646023 \\
\hline & rs7144276 & Intron & Chr14 & 37144607 & $\mathrm{~A} / \mathrm{t}$ & 0.198 & 0.543028 \\
\hline
\end{tabular}

${ }^{\mathrm{a}}$ Uppercase letters denote the more frequent allele in the control samples. SNP, single nucleotide polymorphism; MAF, minor allele frequency; P-HWE, P-value for Hardy-Weinberg equilibrium.

difference between the NSCL/P and control groups $(\mathrm{P}=0.007$, 0.008, 0.001 and 0.003, respectively). For PAX9, there was a significant difference in the allele frequency of rs17176643 between the NSCL/P and control groups $(\mathrm{P}=0.0199)$, but not a significant difference after correction with 10,000 permutations $(\mathrm{P}=0.1349)$. The dominant model and Cochran-Armitage trend test of rs17176643 remained significant between the NSCL/P and control groups ( $\mathrm{P}=0.030$ and 0.049 , respectively). Our results indicate that the MSX1 gene does not influence susceptibility to NSCL/P.

Haplotype analysis. For IRF6, the Haploview program revealed rs2073485, rs2235371, rs2013196, rs7552506, rs2236909, rs861019, rs861020 and rs3753518, are positioned in the same LD block (Fig. 1A). Haplotype analysis of polymorphisms in the IRF6 gene revealed SNP combinations associated with the risk of NSCL/P (Table III). The A-T-C-G-G-A-G-C and $\mathrm{G}-\mathrm{C}-\mathrm{C}-\mathrm{G}-\mathrm{A}-\mathrm{A}-\mathrm{A}-\mathrm{C}$ haplotypes showed an increased risk of NSCL/P following correction with 10,000 permutations $(\mathrm{P}=0.0109$ and 0.0036 , respectively). In MSX1, rs1042484 and rs12532 were in the same LD block (Fig. 1B). The results of the haplotype analysis showed no statistical differences between the NSCL/P and control groups. For PAX9, rs2236007, rs2295218 and rs8004560 were in the same LD block (block 1), while rs11156926, rs10131337 and rs7144276 were in block 2 (Fig. 1C, Table III). No difference at block 1 or 2 between the NSCL/P and control groups was observed.

Gene-gene interaction analysis. A logistic regression model was constructed to analyze the 4 SNPs in IRF6 (rs2073485, rs2235371, rs2236909 and rs861020) and 1 SNP in PAX9 (rs17176643). The combinations of rs2073485 (GG) and rs17176643 (aC+CC), rs2235371 (CC) and rs17176643 (aC+CC), and rs2236909 (aG+aa) and rs17176643 (aC+CC), were significantly associated with NSCL/P following Bonferroni corrections (Table IV).

\section{Discussion}

In the present study, an association with NSCL/P was demonstrated for the SNPs rs2073485, rs2235371, rs2236909 and rs861020, in the IRF6 gene. Haplotype analysis supported these findings. We also found that there was a marginally significant association between NSCL/P and rs17176643 in the PAX9 gene. Moreover, a logistic regression model showed that gene-gene interactions between IRF6 and PAX9 increased the risk of NSCL/P.

Previous studies have shown that variations in IRF6 are responsible for $12 \%$ of the genetic contribution to cleft lip or palate and a significant association was demonstrated between NSCL/P and the rs235371 polymorphism in IRF6, which was also observed in certain populations (21). However, this finding remains controversial in the Chinese population. In the present study, the results of allelic frequencies and a dominant model analysis showed a positive association between the rs235371 polymorphism and NSCL/P. Jia et al reported that rs2073485 polymorphism was associated with NSCL/P in Chinese patients (22), which was consistent with our results. To the best of our knowledge, the relationships demonstrated in the present study between rs2236909 and rs861020 with 


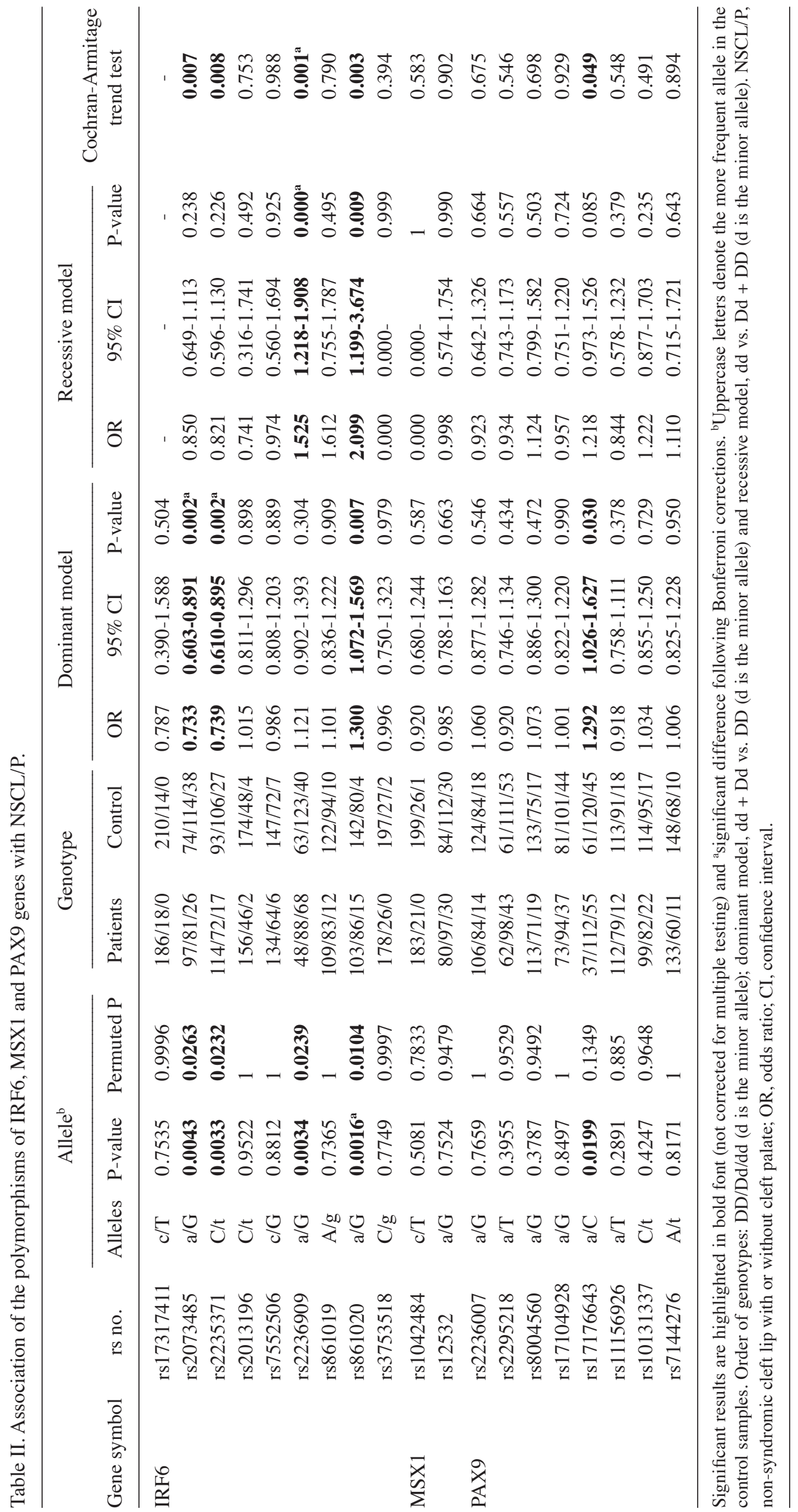


Table III. Haplotype analysis of IRF6, MSX1 and PAX9 gene polymorphisms.

\begin{tabular}{|c|c|c|c|c|c|c|c|}
\hline \multirow[b]{2}{*}{ Gene } & \multirow[b]{2}{*}{ Haplotype } & \multicolumn{2}{|c|}{ Frequencies } & \multirow[b]{2}{*}{ OR } & \multirow[b]{2}{*}{$(95 \% \mathrm{CI})$} & \multirow[b]{2}{*}{ Nominal P } & \multirow[b]{2}{*}{ Permuted P } \\
\hline & & Case & Control & & & & \\
\hline \multirow[t]{5}{*}{ IRF6 } & A-T-C-G-G-A-G-C & 0.26110 & 0.35400 & 1 & Reference & $0.002493^{a}$ & 0.0109 \\
\hline & G-C-C-G-A-A-A-C & 0.28820 & 0.19250 & 2.03 & $1.401-2.94$ & $0.001291^{\mathrm{a}}$ & 0.0036 \\
\hline & G-C-C-G-A-G-G-C & 0.26110 & 0.25220 & 1.404 & $0.9784-2.013$ & 0.8237 & 1 \\
\hline & A-C-C-C-G-A-G-G & 0.06404 & 0.06637 & 1.308 & $0.7327-2.336$ & 0.8638 & 1 \\
\hline & G-C-T-C-G-A-G-C & 0.12320 & 0.12390 & 1.348 & $0.8563-2.121$ & 0.9358 & 1 \\
\hline \multirow[t]{3}{*}{ MSX1 } & $\mathrm{T}-\mathrm{G}$ & 0.62990 & 0.61950 & 1 & Reference & 0.7524 & 0.9442 \\
\hline & C-A & 0.05147 & 0.06195 & 0.8171 & $0.4527-1.475$ & 0.5081 & 0.7935 \\
\hline & $\mathrm{T}-\mathrm{A}$ & 0.31860 & 0.31860 & 0.9836 & $0.735-1.316$ & 0.9989 & 1 \\
\hline \multicolumn{8}{|l|}{ PAX9 } \\
\hline \multirow[t]{3}{*}{ Block 1} & G-A-G & 0.4530 & 0.47990 & 1 & Reference & 0.4136 & 0.9708 \\
\hline & G-T-A & 0.2673 & 0.24110 & 1.175 & $0.8432-1.637$ & 0.3894 & 0.9461 \\
\hline & A-T-G & 0.2772 & 0.26560 & 1.106 & $0.7992-1.53$ & 0.7181 & 0.9996 \\
\hline \multirow[t]{4}{*}{ Block 2} & T-T-A & 0.3062 & 0.27950 & 1 & Reference & 0.4242 & 0.9686 \\
\hline & A-C-A & 0.2493 & 0.27690 & 0.8217 & $0.5716-1.181$ & 0.3336 & 0.9066 \\
\hline & T-C-T & 0.2005 & 0.19330 & 0.9468 & $0.638-1.405$ & 0.8237 & 0.9997 \\
\hline & T-C-A & 0.2408 & 0.24110 & 0.912 & $0.6281-1.324$ & 0.9517 & 1 \\
\hline
\end{tabular}

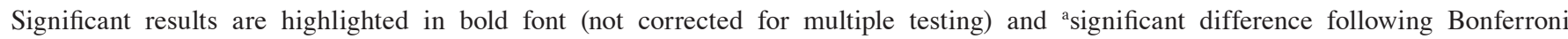
corrections. OR, odds ratio and CI, confidence interval; P, P-value.

Table IV. Results of gene-gene interactions using a logistic regression method.

\begin{tabular}{|c|c|c|c|c|c|c|}
\hline \multirow[b]{2}{*}{ IRF6 $^{\mathrm{a}}$} & \multirow[b]{2}{*}{ PAX9 $9^{a}$} & \multicolumn{2}{|c|}{ Frequencies } & \multirow[b]{2}{*}{ OR } & \multirow[b]{2}{*}{$95 \% \mathrm{CI}$} & \multirow[b]{2}{*}{ P-value } \\
\hline & & Case & Control & & & \\
\hline rs2073485 & rs 17176643 & & & & & \\
\hline $\mathrm{aG}+\mathrm{aa}$ & $\mathrm{CC}$ & 0.073529 & 0.163717 & 1 & Reference & \\
\hline GG & $\mathrm{CC}$ & 0.107843 & 0.106195 & 2.261 & $0.983-5.203$ & 0.055 \\
\hline $\mathrm{aG}+\mathrm{aa}$ & $\mathrm{aC}+\mathrm{CC}$ & 0.450980 & 0.508850 & 1.973 & $1.020-3.816$ & 0.043 \\
\hline GG & $\mathrm{aC}+\mathrm{CC}$ & 0.367647 & 0.221239 & 3.700 & $1.840-7.440$ & $0.000^{b}$ \\
\hline rs2235371 & rs 17176643 & & & & & \\
\hline $\mathrm{Ct}+\mathrm{tt}$ & $\mathrm{CC}$ & 0.058824 & 0.141593 & 1 & Reference & \\
\hline $\mathrm{CC}$ & $\mathrm{CC}$ & 0.122549 & 0.128319 & 2.299 & $0.980-5.390$ & 0.056 \\
\hline $\mathrm{Ct}+\mathrm{tt}$ & $\mathrm{aC}+\mathrm{CC}$ & 0.377451 & 0.446903 & 2.033 & $0.983-4.205$ & 0.056 \\
\hline $\mathrm{CC}$ & $\mathrm{aC}+\mathrm{CC}$ & 0.441176 & 0.283186 & 3.750 & $1.795-7.835$ & $0.000^{b}$ \\
\hline rs2236909 & rs 17176643 & & & & & \\
\hline GG & $\mathrm{CC}$ & 0.044118 & 0.079646 & 1 & Reference & \\
\hline $\mathrm{aG}+\mathrm{aa}$ & $\mathrm{CC}$ & 0.137255 & 0.190265 & 3.433 & $1.391-8.474$ & 0.007 \\
\hline GG & $\mathrm{aC}+\mathrm{CC}$ & 0.191176 & 0.199115 & 1.387 & $0.607-3.170$ & 0.438 \\
\hline $\mathrm{aG}+\mathrm{aa}$ & $\mathrm{aC}+\mathrm{CC}$ & 0.627451 & 0.530973 & 7.002 & $3.154-15.543$ & $0.000^{b}$ \\
\hline rs861020 & rs 17176643 & & & & & \\
\hline GG & $\mathrm{CC}$ & 0.093137 & 0.163717 & 1 & Reference & \\
\hline $\mathrm{aG}+\mathrm{aa}$ & $\mathrm{CC}$ & 0.088235 & 0.106195 & 1.045 & $0.462-2.367$ & 0.915 \\
\hline GG & $\mathrm{aC}+\mathrm{CC}$ & 0.406863 & 0.464602 & 1.398 & $0.739-2.646$ & 0.303 \\
\hline $\mathrm{aG}+\mathrm{aa}$ & $\mathrm{aC}+\mathrm{CC}$ & 0.411765 & 0.265487 & 2.177 & $1.132-4.184$ & 0.020 \\
\hline
\end{tabular}

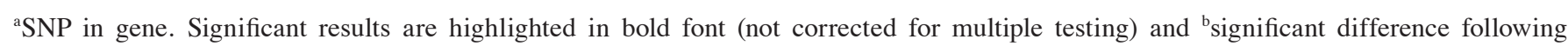
Bonferroni corrections. SNP, single nucleotide polymorphism; OR, odds ratio and CI, confidence interval. 
A

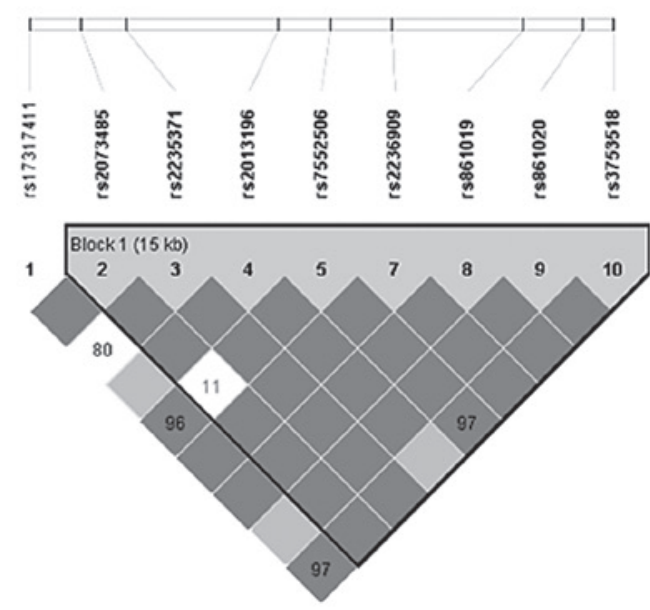

B

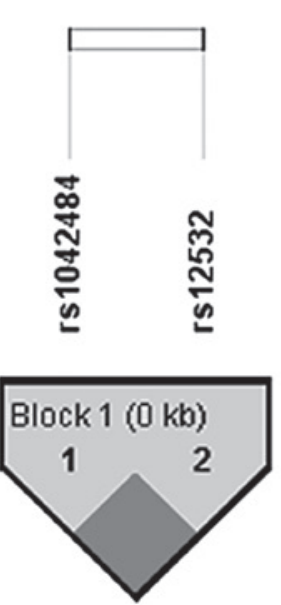

C

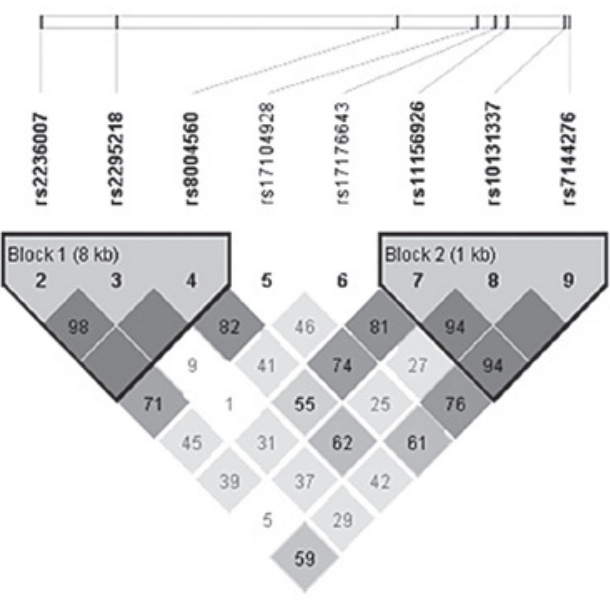

Figure 1. Linkage disequilibrium (LD) pattern for (A) IRF6, (B) MSX1 and (C) PAX9 genes. The haplotype block structure, as exhibited by Haploview is shown. Values within each diamond are the pairwise correlation between the single nucleotide polymorphisms (measured as $\mathrm{D}^{\prime}$ ). The diamond without a number corresponds to $\mathrm{D}^{\prime}=1$. Shading shows the magnitude and significance of pairwise $\mathrm{LD}$, with a black-to-white gradient reflecting higher to lower $\mathrm{D}^{\prime}$ values.

NSCL/P, have not been previously observed. In this study, the allele frequency of rs2236909 in the NSCL/P group showed significant differences from that of the control group. The recessive model and the Cochran-Armitage trend test identified the A variant as a risk factor for NSCL/P. Huang et al demonstrated that there was no significant difference between the NSCL/P and control groups at rs2013162; however, there was a significant difference at rs2235375 (23). These SNPs are located in the same LD block as rs2236909 in the HapMap CHB database. However, the samples in the study by Huang et al were obtained from subjects in West China, while the samples in our study were obtained primarily from patients in North China, which may explain the observed differences. Our results also showed that there was a significant difference in the allele frequency of rs861020 between the NSCL/P and control groups. The dominant and recessive models, as well as the Cochran-Armitage trend test showed differences between the NSCL/P and control groups, although these differences were not significant following Bonferroni corrections. These results suggest that the common A variant is a potential risk factor for NSCL/P in the Chinese population. Other SNPs located in the same LD block as rs861020 in the HapMap CHB database, have not been previously studied. Pegelow et al suggested that the A allele of rs861019 and the $\mathrm{G}$ allele of SNP rs7552506 showed an association with cleft lip and palate in Swedish families (24); however, no significant association was observed in the present study. This may be due to the use of different geographical populations, sample sizes or study methods (family-based versus a case-control study).

MSX1 mutations have been identified in $2 \%$ of cases of NSCL/P and should be considered for genetic counseling. Variants such as CA polymorphisms, A34G, P147Q, G110G, C565T, M37L and G267A, are associated with NSCL/P in various populations $(12,25,26)$; however, this finding remains controversial. In the present study, the case-control analysis using tag SNPs (rs1042484 and rs12532) showed no statistical differences in the allelic frequency or haplotype between the NSCL/P and control groups, which is consistent with the data of Huang et al (13). PAX9 is important in the development of the teeth and lips. Ichikawa et al demonstrated that a heterozygous missense mutation in PAX9 (640A>G, S214G) was linked to the susceptibility for NSCL/P in the Japanese population (16). However, the remaining SNPs in PAX9 did not have a significant association with NSCL/P (17). To the best of our knowledge, a study of PAX9 tag SNPs in the Chinese population has not been previously conducted. In the present study, the allele frequency of rs17176643 was different between the NSCL/P and control groups and this difference was marginally significant following Benjamini-Hochberg corrections $(\mathrm{P}=0.075)$. The dominant model and Cochran-Armitage trend test implied that the common A variant may be a risk factor of NSCL/P in a Chinese population. Lee et al indicated that a haplotype with three SNPs (rs2073247, rs17104928 and rs17176643) showed a significantly increased association with NSCL/P (17).

Interactions between IRF6, MSX1 and PAX9 are important for the development of dentition (27). However, interactions between IRF6 and PAX9 do not contribute to human tooth agenesis (28). The relationship between these interactions and NSCL/P remains unknown. In the present study, a logistic regression model was used to explore gene-gene interactions and their involvement in NSCL/P. The model was based on the combinations of two SNPs, which were significantly or marginally different between the NSCL/P and control groups. The combinations of rs2073485 (GG) and rs17176643 (aC+CC), rs2235371 (CC) and rs17176643 (aC+CC), and rs2236909 (aG+aa) and rs17176643 (aC+CC), significantly increased the risk of NSCL/P, which suggests that these gene-gene interactions are critical in susceptibility to NSCL/P in our study population. The underlying molecular mechanisms of interaction between IRF6 and PAX9 requires further clarification.

In conclusion, regardless of the limitations of a small sample size, the present study demonstrated an association of rs2073485, rs2235371, rs2236909 and rs861020 in the IRF6 gene with NSCL/P in Chinese patients. Haplotype analysis of the gene supported these findings. We also identified that 
gene-gene interactions between IRF6 and PAX9 may be important in the susceptibility to NSCL/P. Additional studies are required to clarify the associations between IRF6, MSX1 and PAX9 with NSCL/P.

\section{Acknowledgements}

The authors thank all the participants who donated samples for this study. This study has been supported by the National Natural Science Foundation of China (grant no. 30901569).

\section{References}

1. Dixon MJ, Marazita ML, Beaty TH and Murray JC: Cleft lip and palate: understanding genetic and environmental influences. Nat Rev Genet 12: 167-178, 2011.

2. Vieira AR: Genetic and environmental factors in human cleft lip and palate. Front Oral Biol 16: 19-31, 2012.

3. Zucchero TM, Cooper ME, Maher BS, et al: Interferon regulatory factor 6 (IRF6) gene variants and the risk of isolated cleft lip or palate. N Engl J Med 351: 769-780, 2004.

4. Wu T, Liang KY, Hetmanski JB, et al: Evidence of gene-environment interaction for the IRF6 gene and maternal multivitamin supplementation in controlling the risk of cleft lip with/without cleft palate. Hum Genet 128: 401-410, 2010.

5. Scapoli L, Palmieri A, Martinelli M, et al: Strong evidence of linkage disequilibrium between polymorphisms at the IRF6 locus and nonsyndromic cleft lip with or without cleft palate, in an Italian population. Am J Hum Genet 76: 180-183, 2005.

6. Park JW, McIntosh I, Hetmanski JB, et al: Association between IRF6 and nonsyndromic cleft lip with or without cleft palate in four populations. Genet Med 9: 219-227, 2007.

7. Brito LA, Bassi CF, Masotti C, et al: IRF6 is a risk factor for nonsyndromic cleft lip in the Brazilian population. Am J Med Genet A 158A: 2170-2175, 2012

8. Shi J, Song T, Jiao X, Qin C and Zhou J: Single-nucleotide polymorphisms (SNPs) of the IRF6 and TFAP2A in non-syndromic cleft lip with or without cleft palate (NSCLP) in a northern Chinese population. Biochem Biophys Res Commun 410: 732-736, 2011.

9. Pan Y, Ma J, Zhang W, et al: IRF6 polymorphisms are associated with nonsyndromic orofacial clefts in a Chinese Han population. Am J Med Genet A 152A: 2505-2511, 2010.

10. Nugent $P$ and Greene RM: MSX-1 gene expression and regulation in embryonic palatal tissue. In Vitro Cell Dev Biol Anim 34: $831-835,1998$

11. Nakatomi M, Wang XP, Key D, et al: Genetic interactions between Pax9 and Msx1 regulate lip development and several stages of tooth morphogenesis. Dev Biol 340: 438-449, 2010.

12. Salahshourifar I, Halim AS, Wan Sulaiman WA, et al: Contribution of MSX1 variants to the risk of non-syndromic cleft lip and palate in a Malay population. J Hum Genet 56: 755-758, 2011.
13. Huang YQ, Ma J, Ma M, et al: Association between MSX1 variants and oral clefts in Han Chinese in western China. DNA Cell Biol 30: 1057-1061, 2011.

14. Suazo J, Santos JL, Jara L and Blanco R: Parent-of-origin effects for MSX1 in a Chilean population with nonsyndromic cleft lip/palate. Am J Med Genet A 152A: 2011-2016, 2010.

15. Perry GH, Verrelli BC and Stone AC: Molecular evolution of the primate developmental genes MSX1 and PAX9. Mol Biol Evol 23: 644-654, 2006.

16. Ichikawa E, Watanabe A, Nakano Y, et al: PAX9 and TGFB3 are linked to susceptibility to nonsyndromic cleft lip with or without cleft palate in the Japanese: population-based and family-based candidate gene analyses. J Hum Genet 51: 38-46, 2006.

17. Lee JK, Park JW, Kim YH and Baek SH: Association between PAX9 single-nucleotide polymorphisms and nonsyndromic cleft lip with or without cleft palate. J Craniofac Surg 23: 1262-1266, 2012.

18. Sull JW, Liang KY, Hetmanski JB, et al: Maternal transmission effects of the PAX genes among cleft case-parent trios from four populations. Eur J Hum Genet 17: 831-839, 2009.

19. Matalova E, Fleischmannova J, Sharpe PT and Tucker AS: Tooth agenesis: from molecular genetics to molecular dentistry. J Dent Res 87: 617-623, 2008.

20. Slayton RL, Williams L, Murray JC, et al: Genetic association studies of cleft lip and/or palate with hypodontia outside the cleft region. Cleft Palate Craniofac J 40: 274-279, 2003.

21. Zucchero TM, Cooper ME, Maher BS, et al: Interferon regulatory factor 6 (IRF6) gene variants and the risk of isolated cleft lip or palate. N Engl J Med 351: 769-780, 2004.

22. Jia ZL, Li Y, Li L, et al: Association among IRF6 polymorphism, environmental factors, and nonsyndromic orofacial clefts in western china. DNA Cell Biol 28: 249-257, 2009.

23. Huang Y, Wu J, Ma J, et al: Association between IRF6 SNPs and oral clefts in West China. J Dent Res 88: 715-718, 2009

24. Pegelow M, Peyrard-Janvid M, Zucchelli M, et al: Familial non-syndromic cleft lip and palate - analysis of the IRF6 gene and clinical phenotypes. Eur J Orthod 30: 169-175, 2008.

25. Tongkobpetch S, Siriwan P and Shotelersuk V: MSX1 mutations contribute to nonsyndromic cleft lip in a Thai population. J Hum Genet 51: 671-676, 2006.

26. Butali A, Mossey PA, Adeyemo WL, et al: Genetic studies in the Nigerian population implicate an MSX1 mutation in complex oral facial clefting disorders. Cleft Palate Craniofac J 48: 646-653, 2011.

27. Boeira BR Jr and Echeverrigaray S: Dentistry and molecular biology: a promising field for tooth agenesis management. Tohoku J Exp Med 226: 243-249, 2012.

28. Vieira AR, Cooper ME, Marazita ML, Oriolo IM and Castilla EE: Interferon regulatory factor 6 (IRF6) is associated with oral-facial cleft in individuals that originate in South America. Am J Med Genet A 143A: 2075-2078, 2007. 